\title{
The Design of Video Surveillance System Based on IEEE802.11n
}

\section{Protocol}

\author{
Qing-Liang SHAO ${ }^{1, a}$, Zi-Yang YE ${ }^{1, b}$, Yue-run WANG ${ }^{1, C}$ \\ ${ }^{1}$ Shenyang Aerospace University, Shenyang City, LiaoNing Prov, China \\ ashaoql@sau.edu.cn, byeziyang1992@163.com, cy841921983@qq.com
}

Key words: video capture, STM32, IEEE802.11n, RT5350 chip

Abstract. A design of system of video monitoring cars based on IEEE802.11n protocol. STM32 chip as the control core, image data gathered by free driver USB camera, was used to compression coding, which was sent to video receiver by the use of RT5350 wireless router module with WIFI. Then the user could send control command according to the situation to change the car running status to realize the spot real-time monitoring. The text showed that this system, with clear image acquisition, stable propagation effects and instant effective control, has a good application prospect.

\section{Introduction}

With the rapid development of network and multimedia technology, the application of the video monitoring system based on embedded technology is becoming more and more popular. The design of embedded wireless video data acquisition system based on RT5350 chip is proposed in Reference $^{[1]}$, which configures the system hardware in detail and realizes the aim of the web video monitoring. S3C6410 is taken as the core processor in Reference ${ }^{[2]}$. The video transmission scheme based on IEEE $802.11 \mathrm{~g}$ protocol is designed by collecting image information of USB camera. Moreover, in Reference ${ }^{[3]}$, a large number of researches on acquisition and coding of video were carried out, and Android client software is specially designed to accept the data, which makes the system with a better mobility. At present, the majority of video monitoring systems are lack of liquidity and flexibility, without the car system configuration and the design of the pan-fit device of camera. This paper integrates the design idea of predecessors, and then put forward the design of video monitoring system based on IEEE802.11n protocol. And this design scheme successfully implement video monitoring and wireless remote control, and has the advantages such as simple structure, low cost, stable transmission, etc.

\section{The design of hardware}

Hardware platform of video monitoring car system is mainly composed of STM32F103 microcontroller, wireless router module based on RT5350 chip. The output image system is consisted by USB camera-free dive with the MJPEG format, two 3-wire steering gears, double L298N driver module, and so on. The general block diagram of hardware is shown in Figure 1. The wireless routing module applied by the system has reserved TTL serial port, TTL serial port is a kind of communication serial port and it has three lines: TX is used to send data, RX is used to receive data and GND is used to lead the ground. The TTL serial port of wireless routing module was connected to the serial port of STM32 single chip microcomputer and the software writing and processing was conducted in RT5350 chip. And the wireless routing module can communicate with the single chip microcomputer. RT5350 wireless routing module is equipped with an antenna, 
which has the effects of enhancing emission signal and expanding signal coverage range.

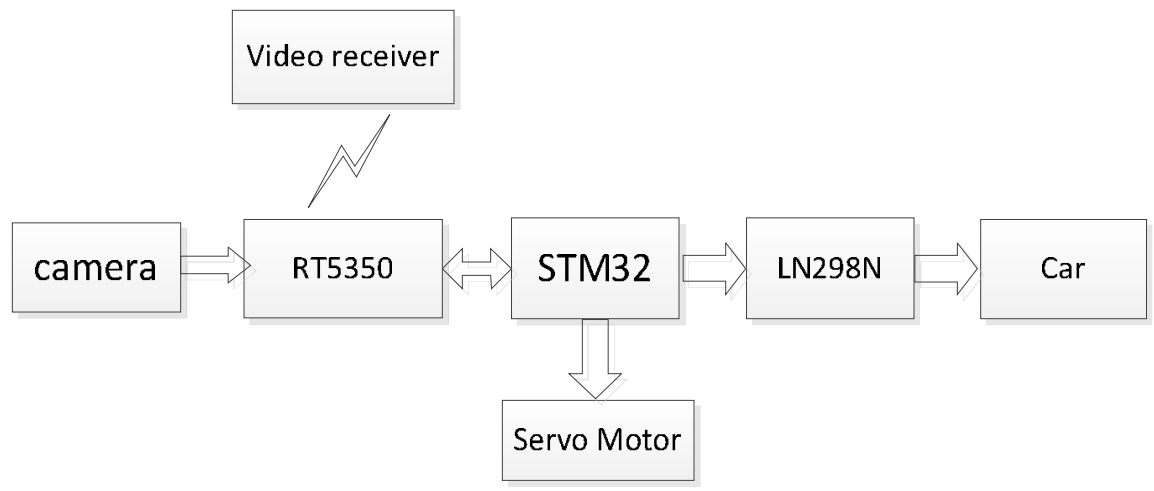

Fig. 1 The block diagram of hardware

USB camera is connected with HOST USB interface of the wireless routing module via an USB line. USB line applies serial mode to transport data, it has wide transmission bandwidth and can totally satisfy bandwidth required for transporting video. The three lines of 3 line rudder are GND line, VCC line and DATA line, the DATA line is used to control the rotation angle of rudder. This design applies 2 rudders to better control the rotation from left to right and up to down of the camera, which can let the litter car monitor the situation around with all directions in the narrow area.

The PA.0-PA.7 I0 interface of STM32 microcontroller connects with IN1-IN8 pin of double L298N driver module. Microcomputer controls the size of electrical level of its output pin (I0 interface) so that the motor driver chip drive motor forward rotation or reversal to realize the car to advance and withdraw.

\section{The design of software}

Software design mainly includes two parts: one is video transceiver driver of RT5350 wireless router module and WIFI setup procedure. The other one is the steering gear control procedures and motor drive control procedures based on WIFI. The former is burned to the RT5350 chip under the Linux environment. The latter is burned to the STM32 microcontroller under the Keil environment. The wireless router module receive images information from the camera by a USB line, and image coding and compression are conducted on these information by MJPG-streamer software, finally images which have been converted to binary data are sent to WIFI channel ${ }^{[4]}$. Upper computer decodes after accepting data and displays the image. When the rate of transmitting image is more than 24 frames per second, what the people see is a dynamic video rather than a static picture. Users can operate the related button on the upper computer to control the motion state of car and the orientation of the camera. The overall software flow diagram of system is shown in Figure 2. 

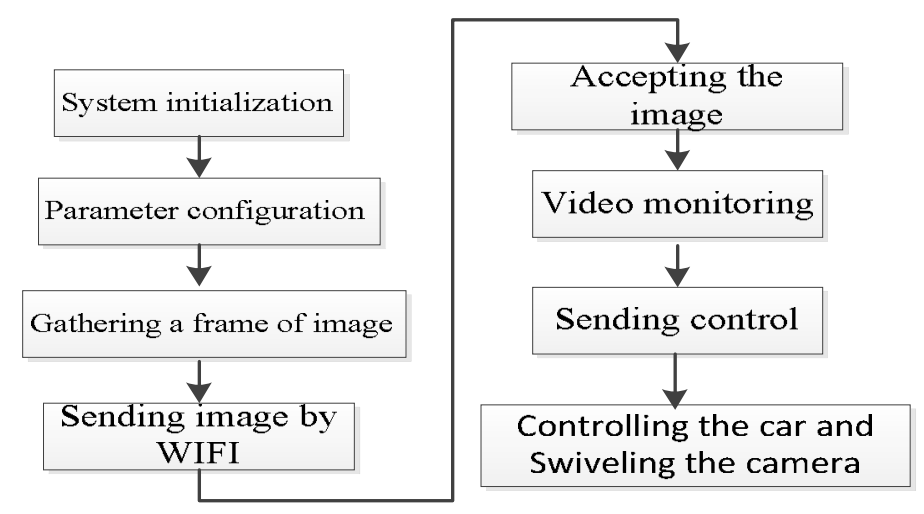

Fig. 2 software flow chart

\section{The software configuration of RT5350 wireless router module}

RT5350 chip is a programmable system chip on the wireless chip with high quality, small size and low cost, which provides the superior wireless router solution ${ }^{[5]}$. Method of RT5350 wireless router module configuration is that the Openwrt system of open source is brushed into the chip, and then various programs and drivers are installed on it. In this way, users can take the route as the platform, and freely loads the devices such as USB camera, network CARDS and so on. Openwrt is a highly modular, highly automated embedded Linux system, provides a completely writable file system and package management, furthest reduces the threshold of the embedded Linux development with a simple and easy way. It allows the use software packages to customize the embedded-type devices, which makes it suitable for most applications and achieve more functions. Openwrt system brushed into the open source must be conducted under the environment of Linux compilation. So a virtual machine is created in the general computer, then the Linux system is installed. After installation, the Linux system is configured, and two services ftp v ssh are started. These two services mainly are to configure and start the network Settings of Linux operating system of host. Then, Openwrt open source system is compiled by downloading configuration.

Before, three related tools and libraries need to be installed firstly.

(1) Install SVN tool for downloading Openwrt source code.

\$ sudo apt-get install subversion

(2) Install git tools.

$\$$ sudo apt-get install git-core

(3) Install reliant library file.

\$ sudo apt-get install gec g++ binutils patch bzip2 flex bison make autoconf gettext texinfo unzip sharutils ncurses-term zlib1g-dev libncurses5-dev gawk

A new Openwrt folder is created, and the source code is downloaded by using SVN.

$\$$ mkdir Openwrt

$\$$ cd Openwrt/

\$svn co svn://svn. Openwrt.org/ Openwrt/trunk

After downloading the Openwrt source code, in order to make the Openwrt support more software, it is needed to update and install the software in other sources.:

$\$$ cd trunk

$\$ . /$ scripts/feeds update $-\mathrm{a}$

$\$ . /$ scripts/feeds install -a

Everything is ready, and began to compile. As compiling, firstly, make menuconfig is executed to 
configure settings. Finally, make $\mathrm{V}=99$ is executed, and lengthy compilation begins. After the compilation is completed, mirroring is generated under the source code directory bin. Among them, Openwrt-ramips-rt305x-mpr-a2-squashfs-sysupgrade.bin is the mirroring used by burning, which is started as burning to wireless router module. Like this, the Openwrt system completes the transplantation.

The wireless routing module can emit WIFI signal when the AP mode is selected. The so-called AP mode is that WIFI signal is given out for others to connect. The users just need modify the configuration file in the Openwrt system.

The frequency band of WIFI signal is given out from wireless router module which is successfully configured, which adopts the IEEE802.11n standard. The transmission rate of standard theory can reach $320 \mathrm{MB} / \mathrm{s} \sim 600 \mathrm{MB} / \mathrm{s}$, which fully meet the requirements of system for video transmission and real-time image monitoring.

Wireless router module need support the camera, and must be configured the camera driver. Because it is the USB camera-free drive, must be configured UVC driver. The make menuconfig is executed in the top-level directory of source code, and then the choice is made as follows.

Device Drivers -->

Multimedia devicces -->

$<^{*}>$ Video For Linux

Video Capture Adapters -->

$<*>$ USB Video Class(UVC)

After configuring the UVC driver and then MJPG-streamer software is loaded. MJPG-streamer is a software for video data acquisition, video data format conversion and video data transmission. Firstly, Makefile file is modified under the input UVC directory so as to meet the compilation command. Then execute the order make CC =arm-Linux-GCC and compile it. Copy the executable file MJPG streamer after compilation under the target file path to bin folder under the development board root directory, so far, the whole configuration process is completed. Next, insert the USB camera-free drive, and RT5350 wireless router module can conduct video image transmission.

\section{servo-driven program}

I/O port of STM32 microcontroller connects to the cable of steering engine, PWM signal is input to microcomputer and sent to steering engine, then input to the steering gear, and then the rotation angel of the steering engine is controlled. The control signal cycle of steering engine is the pulse width modulation (PWM) signal with a cycle of $20 \mathrm{MS}$, and the pulse width is from 0.5 to 2.5 MS, and the corresponding steering wheel position is $0-180^{\circ}$ with a linear change. Providing it a certain pulse width, the output shaft will keep a corresponding angle, no matter how the outside torque changes. There is a benchmark circuit inside of the steering engine. It can generates the benchmark signal with a cycle of $20 \mathrm{MS}$ and the width of $1.5 \mathrm{MS}$. In addition, there is a comparator, it compares the external signal with the benchmark signal, judges the direction and size, and then generates the rotation signal of steering engine.

\section{DC motor-driven program}

DC Motor rotation mainly depends on double L298N drive. The change of steering engine drive $\mathrm{H}$ port voltage is caused by the change of high and low level of microcontroller PA port, which can control the forward/reversal rotation of DC Motor. 


\section{The tests of system}

After the related hardware and software design of this system is completed, upper computer program need to be compiled for video monitoring and wireless remote control. Video acceptance and control interface are shown in Figure 3.

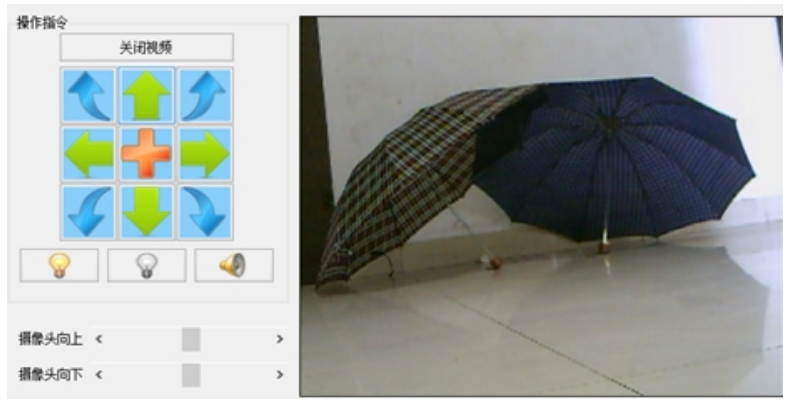

Fig. 3 Video acceptance and control interface

Video acceptance and control interface are shown in Figure 3. The development environment of upper computer program is VB6.0, and it is needed to call the Active control and Winsock control for completing the exploitation of upper computer program. The control port number of upper computer is 2001, and the control address is 192.168.1.1. Instruction set has five keys such as forward, backward, left, right, stop, corresponding to W, S, A, D blank space keys on the keyboard. A string of binary number can be sent out by WIFI when pressing one of the keys each time. The lower computer accepts a binary number to change the running state of car. The steering engine rotation angle of lower computer can be changed by the slide of camera slip sheet of upper computer interface in order to control the rotation of the camera.

\section{Summary}

This system better realize the video monitoring and remote wireless control of the intelligent car. The monitoring pictures are transmitted by wireless router module with the embedded IEEE802.11 $\mathrm{n}$ protocol, which has the characteristics such as fast transmission speed, stable transmission, etc. The successful implementation of the system mainly lies in the construction of wireless local area network with the WiFi technology. In the built wireless local area network, camera module collects video information, relatively remote wireless transmission is realized through the AP mode, and the users performs the eal-time monitoring in the receiver. This system provides a complete WIFI video transmission scheme, which is more suitable for the increasingly fiery development prospects of Internet of Things.

\section{References}

[1] YUAN Xin-di,LI Qiu-sheng. Embedded wireless video data acquisition system based on RT5350 [J]. Journal of Anhui University,2014,38(6):60-65

[2] ZHAN Yi-jia, DENG Ben-zai, CAO Yu-jie. Design of a wireless video monitoring system based on S3C6410 [J]. Electronic Design Engineering,2015.23(4):172-175

[3] Chen Heng-xin, Lin Wei, Zhang Qin-yu. Design of Mobile Video Surveillance System Based on Mjpg-streamer [J]. Computer Measurement \& Control,2014.22(11):3597-3599

[4] WANG Yu-yang, YANG Yi, HAN Zhi. WiFi Monitoring Smart Robot in Android Devices [J]. Computer \& Digital Enginneering,2014.42(12):2411-2411

[5] LI Zhi-shan, SU Wei-ji. Design of Linux router for HD video transmission [J]. COMPUTER 
ENNGINEERING AND DESIGN， 2015,36(1):127-129 